\section{Microfracture for treatment of knee cartilage defects in children and adolescents}

\author{
Gian M. Salzmann, Bert-Ram Sah, \\ Hagen Schmal, Philipp Niemeyer, \\ Norbert P. Sudkamp
}

Department of Orthopaedic and Trauma Surgery, University Medical Center, Albert-Ludwigs University Freiburg, Freiburg, Germany

\begin{abstract}
Even though operative microfracture is the most frequent method for treatment of limited knee joint cartilage lesions among adults, data about ouctome in children and adolescents are rare. We performed a retrospective chart review and telephone interview to analyze for the clinical outcome following knee joint cartilage defect microfracturing among 10 children. Mean postoperative Lysholm was $92.1 \pm 9.9$ and Tegner was 7.0 \pm 1.9 . Clinical outcome differed across knee joint regions, as well as in dependence of varying pre-operative symptom duration, although this was not significant. Regression analysis did not reveal a significant impact of patient or defect characteristics on clinical outcome. Arthroscopic microfracturing for treatment of limited size symptomatic knee joint cartilage defects among children and adolescents is considered a reasonable surgical option. However, long-term outcome and larger patient cohorts are required.
\end{abstract}

\section{Introduction}

The surgical technique of arthroscopic microfracturing is indicated for treatment of small-and mid-diameter knee joint cartilage lesions. Using awls, blunt holes (microfractures) are punched into the subchondral bone of the debrided chondral lesion. ${ }^{1}$ Hematoma settles within the defective region and a stem cell fraction among this super clot is sought to differentiate into cartilage or cartilage-like tissue over time. It can currently be considered the most frequent treatment modality for symptomatic cartilage defects across the knee joint among adult patients. ${ }^{2}$ Long-term outcome, as described by Steadman and colleagues, is satisfying. ${ }^{3}$ Despite its popularity, there is only minimal evidence in the current literature to describe clinical results following microfracturing among children and adolescents. However, this population presents physiological differences in mesenchymal stem cell (MSC) quanti- ty and activity, ${ }^{4}$ that potentially provide a more potent super clot than in adult patients. Furthermore, less invasive surgery may be particularly indicated in children and younger adolescents. The aim of this study was to report on clinical outcome following knee joint microfracture in the treatment of limited chondral lesions among children and adolescents.

\section{Materials and Methods}

From a large cohort $(\mathrm{n}=560)$, we selected patients under the age of 17 years at the time of surgery who had been treated for knee cartilage defects using the microfracturing technique at our institution between 2001 through 2008. Patients' characteristics (age, gender, body mass index (BMI), symptom duration until surgery, follow-up time, previous surgery) and defect morphology (location, diameter in square centimeter, depth according to the International Cartilage Repair Society, ICRS) ${ }^{5}$ were collected through a review of medical records. Clinical outcome (subjective International Knee Documentation Committee (IKDC) Knee form score, ${ }^{6}$ Lysholm score, ${ }^{7}$ Tegner activity scale, ${ }^{8}$ as well as a pre-and postoperative numeric analog scale (NAS) for pain (NAS-P) and function (NAS-F) with 0 representing no pain and 10 representing maximal imaginable pain) was obtained by telephone interview. All study data were anonymized during the study process. The study was approved by the local institutional review board, and all patients gave their oral consent to the interview.

The surgical approach has been comprehensively described before. ${ }^{9}$ Patients with a clinically clear symptomatology, i.e. adequate cartilage lesion(s) on pre-operative magnectic resonance imaging (MRI), were considered for surgery. The final decision to proceed with microfracturing was made during knee arthroscopy depending on lesion characteristics as well as general intraarticular knee morphology. The indication was given for symptomatic full-thickness chondral defects, without extensive subchondral bony deficiency and lesion size with a maximum diameter of $3 \mathrm{~cm}^{2}$. Statistical analysis was performed using the software package SPSS version 17 (SPSS Inc, Chicago, Illinois, USA). All data were tested for normal distribution using the KolmogorovSmirnov test. Data were later compared using t-tests or Mann-Whitney U and Wilcoxon's signed rank tests. Group data were compared using one-way analysis of variance or KruskalWallis analysis. $\chi^{2}$ test was used to define independence and linear regression analysis was then carried out to evaluate the effects of patient and/or defect characteristics on the final clinical outcome. Unless otherwise stat-
Correspondence: Gian Salzmann, Department of Orthopaedic and Trauma Surgery, University Medical Center, Albert-Ludwigs University Freiburg, Hugstetter Strasse 55, 79106 Freiburg, Germany.

Tel. +49.761.270.24010 - Fax +49.761.270.26160.

E-mail: giansalzmann@yahoo.com

Key words: microfracture, cartilage, knee joint, children, adolescent.

Conflict of interests: the authors report no potential conflict of interests.

Contributions: GS, HS data analysis and preparation of manuscript; B-RS data collection; PN data analysis, preparation of manuscript, surgery of patients; NS data analysis, surgery of patients.

Received for publication: 6 September 2011. Accepted for publication: 20 November 2011.

This work is licensed under a Creative Commons Attribution NonCommercial 3.0 License (CC BYNC 3.0).

(C) Copyright G.M. Salzmann et al., 2012

Licensee PAGEPress, Italy

Pediatric Reports 2012; 4:e21

doi:10.4081/pr.2012.e21

ed, descriptive results are demonstrated as the mean \pm standard deviation. $\mathrm{P}<0.05$ was considered significant for all tests.

\section{Results}

From the available 560 subjects, a total of 454 (81.1\%) were successfully contacted via telephone and completed the interview. Ten patients (2.2\%) fulfilled inclusion criteria as children or adolescents. There were 2 females and 8 males (BMI 21.8 \pm 3.4 ). Mean age at time of surgery was $14.1 \pm 2.3$ (one each aged 9,11 , and 14 years, 5 aged 15 years, 2 aged 16 years) and at time of interwiew $17.7 \pm 2.6$ (follow up $3.5 \pm 1.5$ ) years. Average symptom duration was $12.1 \pm 13.1$ months. A total of 4 subjects had undergone previous surgery at the index knee, 3 subjects reported knee trauma leading to symptoms. All cartilage defects were single lesions (3 medial femur, 2 lateral femur, 2 trochlea, 2 patella, one lateral tibia plateau) with an average defect size of $1.2 \pm 0.80 \mathrm{~cm}^{2}$. There were 3 ICRS $3^{\circ}$ lesions and 7 ICRS $4^{\circ}$ lesions; bony deficiency was no indication against microfracturing in these cases. Lesion etiology was trauma in 5 cases, OD in 3 cases and degeneration in 2 cases.

Mean postoperative Lysholm was $92.1 \pm 9.9$, IKDC $90.4 \pm 8.2$ and Tegner 7.0 \pm 1.9 . NAS-P improved from $8.2 \pm 2.2$ to $2.0 \pm 1.1(\mathrm{P}<0.001)$; 
NAS function changed from $7.9 \pm 1.4$ to $1.7 \pm 0.8$ $(\mathrm{P}<0.001)$. The clinical outcome differed across knee joint regions (best outcome lateral femur, worst outcome patella undersurface) as well as in variations in pre-operative symptom duration (better outcome with shorter symptom duration); this was not significant. Furthermore, there was no significant difference between patients with and without previous surgery $(\mathrm{P}=0.068$; better outcome without previous surgery) or trauma and no trauma $(\mathrm{P}=0.90$; better outcome with previous trauma) leading to knee joint pain. Regression analysis did not show a significant impact of patient or defect characteristics on the clinical outcome.

When compared to the remaining adult subjects $(n=444)$, all clinical outcome parameters were significantly better in children and adolescents (values for adults: Lysholm 70.7 \pm 21.7 $(\mathrm{P}<0.001)$, IKDC 66.5 $\pm 20.6(\mathrm{P}<0.001)$, Tegner $3.9 \pm 1.8 \quad(\mathrm{P}<0.001)$, postoperative NAS-P $2.9 \pm 2.0(\mathrm{P}=0.003)$, and postoperative NAS-F $2.7 \pm 1.7(\mathrm{P}=0.002)$.

\section{Discussion}

Study results show that clinical outcome following microfracture at the knee joint for treatment of cartilage defects among children and adolescents gives a very satisfying clinical outcome at 3.5 years post surgery. While specific patient/defect parameters can affect the outcome, this was not significant, most likely due to the small study group. Compared to the adult subjects treated at our institution, clinical outcome is clearly superior. This assertion does not change when we reduce the adult population to single, diameter-comparable defects (data not shown).

In general, cartilage defect repair among children and adolescents remains a topic of discussion. There is no literary information on the natural history of existing defects among young patients, while Ding and colleagues reported that changes among adult subjects are associated with female sex, age, and body mass index. ${ }^{10}$ According to age and anatomy, defect etiology is commonly either trauma with sheared off osteochondral fragments or underlying osteochondrosis dissecans (OD). There is a heavy body of literature reporting on management of loose joint bodies among young patients but it is not truly conclusive. ${ }^{11}$ Considering that osteochondral loose body fragments are viable, ${ }^{12}$ preservation with reduction and fixation of these fragments should be attempted whenever possible. Symptomatic OD can generally be managed first by drilling procedures. ${ }^{13}$ However, as in the subjects reported here, there remain cases with either chronic cartilage lesions or no given possibility for fragment recovery. For those patients, common operative cartilage defect repair options apply. While there are guidelines for use in adult patients, this treatment is still new territory among children and adolescents as far as reparative/regenerative defect repair is concerned.

Gudas et al. ${ }^{14}$ prospectively reported on 50 children with a mean age of 14.3 years who had either been randomized to treatment by osteochondral autografts (OAT) or arthroscopic microfraturing for osteochondritis dissecans defects in the knee. At a mean 4.2-year follow up, mosaic-type OAT was significantly superior to microfracturing. In contrast to the patients reported here, defect size was considerably larger $\left(3.17 \mathrm{~cm}^{2}\right)$ and symptom duration longer (23.54 months), which may have affected the poorer compared with OAT. We consider $3 \mathrm{~cm} 2$ as the maximum defect size for microfracture treatment while in larger lesions autologous chondrocyte transplantation, as reported with satisfying outcome by Micheli, 15 is clearly favored over OAT at our institution.

The final success of a microfracturing procedure among adult subjects has been shown to be generally dependent on patient age, duration of symptoms, lesion size, BMI, pre-operative activity level, previous surgery and repair cartilage volume. ${ }^{16}$ These factors may also be linked to the quality of the clot that is forming within the defective region (Figure 1). This becomes much more important since it has been reported that clinical symptoms can be related to the quality of repair tissue. 17

It is known that general stem cell quantity and quality is strongly influenced by age and, to a lesser extent, gender and also by underlying individual conditions. ${ }^{18}$ Fehrer and colleagues have previously reported that aged human MSC show a decline in differentiation potential as well as in proliferation rate. In agreement with this, Muschler described a significant agerelated decline in the number of nucleated cells that were harvested (iliac crest) per aspirate for both men and women. ${ }^{19}$ One may speculate that the superior quantity, proliferative activity and differentiation capacity of stem cells in children and adolescents may provide an improved repair tissue. An improved intraarticular tissue morphology in turn may be minimizing symptoms, resulting in very good function. As in Mithoefer's analysis, the symptom duration and previous surgery also affected the clinical outcome in this study cohort. However, the sample size is too small to show a significant impact. However, besides the above reported MSC quality, we consider symptom duration the other major factor to affect clinical outcome. It has been shown in experimental and in clinical studies that existing cartilage lesions may initiate a catabolic intraarticular environment. ${ }^{20,21}$ Joint homeostasis is altered and detrimental cytokines are over-expressed. These sequences are chronic in nature, while existing cartilage lesions may progress. ${ }^{22}$ Cartilage defect repair in an arthritic setting may result in an inferior outcome when compared to unaltered operative conditions. In particular, children and adolescents have a much stronger general tissue repair capacity, which can also be found for joint homeostatis. Chronic defects, longer symptom duration may be much better tolerated and balanced in younger patients.

The limitations of this study are in its retrospective nature and small sample size. Furthermore, no standard MRI documentation

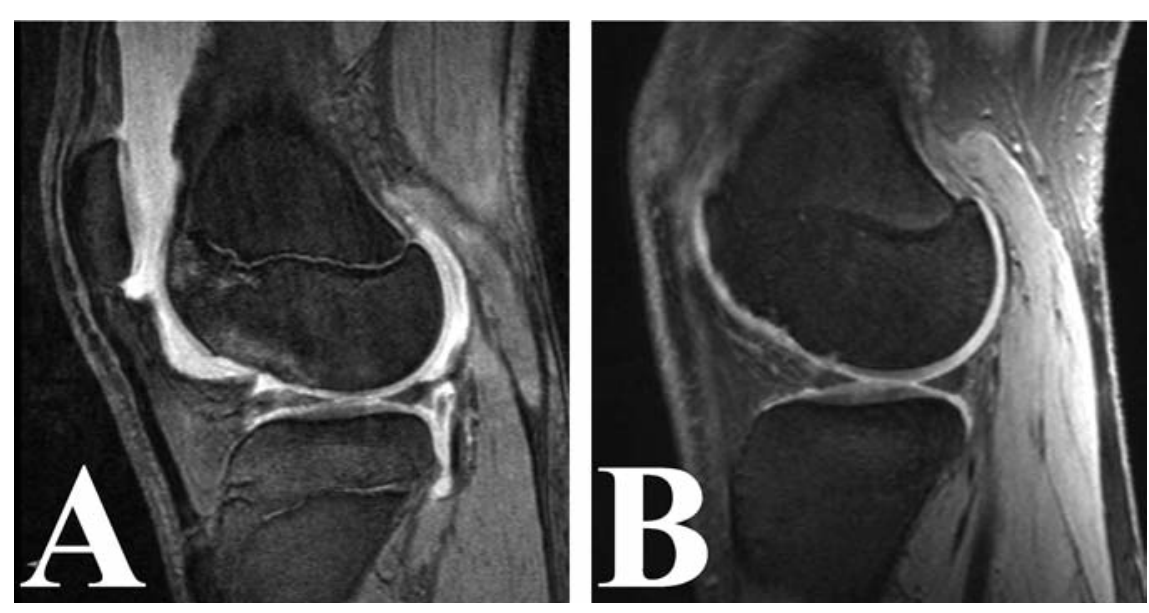

Figure 1. (A) Pre-operative magnetic resonance imaging (MRI) shows large fresh traumatic chondral lesion of the lateral femoral condyle in a 15-year old boy with open physis. (B) 14-month postoperative follow-up MRI regression of bone marrow edema and satisfying cartilage defect fill with well shouldered border integration and almost isointense cartilage signal. Irregularities remain within the subchondral bone. 
is available postoperatively, because all 10 subjects were very satisfied with the procedure result and, therefore, MRI was not clinically indicated. The advantages are to be found in the fact that this is the second study in the current literature to report the results of microfracturing among children and adolescents. Furthermore, the collective of consecutive adult patients for direct internal comparison can be considered quite large and, therefore, representative.

\section{Conclusions}

In conclusion, we consider arthroscopic microfracturing for treatment of limited size symptomatic knee joint cartilage defects among children and adolescents a reasonable surgical option. However, evaluation of longterm outcome in larger patient cohorts is required.

\section{References}

1. Steadman JR, Singleton SB, Briggs KK. Microfracture technique for full-thickness chondral defects: technique and clinical results. . Oper Tech Orthop 1997;7:300-4.

2. Farr J, Cole B, Dhawan A, et al. Clinical Cartilage Restoration: Evolution and Overview. Clin Orthop Relat Res 2011;469: 2696-705.

3. Steadman JR, Briggs KK, Rodrigo JJ, et al. Outcomes of microfracture for traumatic chondral defects of the knee: average 11year follow-up. Arthroscopy 2003;19:47784.

4. Fehrer C, Lepperdinger G. Mesenchymal stem cell aging. Exp Gerontol 2005;40:92630 .
5. van den Borne MP, Raijmakers NJ, Vanlauwe $\mathrm{J}$, et al. International Cartilage Repair Society (ICRS) and Oswestry macroscopic cartilage evaluation scores validated for use in Autologous Chondrocyte Implantation (ACI) and microfracture. Osteoarthritis Cartilage 2007;15:1397-402.

6. Brittberg M, Winalski CS. Evaluation of cartilage injuries and repair. J Bone Joint Surg Am.2003;85:58-69.

7. Lysholm J, Gillquist J. Evaluation of knee ligament surgery results with special emphasis on use of a scoring scale. Am J Sports Med 1982;10:150-4.

8. Tegner Y, Lysholm J. Rating systems in the evaluation of knee ligament injuries. Clin Orthop Relat Res 1985:43-9.

9. Steadman JR, Rodkey WG, Rodrigo JJ. Microfracture: surgical technique and rehabilitation to treat chondral defects. Clin Orthop Relat Res 2001;391:S362-9.

10. Ding C, Cicuttini F, Scott F, et al. Natural history of knee cartilage defects and factors affecting change. Arch Intern Med 2006;166:651-8.

11. Kocher MS, Tucker R, Ganley TJ, Flynn JM. Management of osteochondritis dissecans of the knee: current concepts review. Am J Sports Med 2006;34:1181-91.

12. Pascual-Garrido C, Tanoira I, Muscolo DL, et al. Viability of loose body fragments in osteochondritis dissecans of the knee. A series of cases. Int Orthop 2010;34:827-31.

13. Hayan R, Phillipe G, Ludovic S, et al. Juvenile osteochondritis of femoral condyles: treatment with transchondral drilling. Analysis of 40 cases. J Child Orthop 2009. Epub ahead of press.

14. Gudas R, Simonaityte R, Cekanauskas E, Tamosiunas R. A prospective, randomized clinical study of osteochondral autologous transplantation versus microfracture for the treatment of osteochondritis dissecans in the knee joint in children. $\mathrm{J}$ Pediatr
Orthop 2009;29:741-8.

15. Micheli LJ, Moseley JB, Anderson AF, et al. Articular cartilage defects of the distal femur in children and adolescents: treatment with autologous chondrocyte implantation. J Pediatr Orthop 2006;26:455-60.

16. Mithoefer K, McAdams T, Williams RJ, et al. Clinical efficacy of the microfracture technique for articular cartilage repair in the knee: an evidence-based systematic analysis. Am J Sports Med 2009;37:205363 .

17. Brun P, Dickinson SC, Zavan B, et al. Characteristics of repair tissue in secondlook and third-look biopsies from patients treated with engineered cartilage: relationship to symptomatology and time after implantation. Arthritis Res Ther 2008;10: R132.

18. Leonardi E, Devescovi V, Perut F, et al. Isolation, characterisation and osteogenic potential of human bone marrow stromal cells derived from the medullary cavity of the femur. Chir Organi Mov 2008;92:97103.

19. Muschler GF, Nitto H, Boehm CA, Easley KA. Age- and gender-related changes in the cellularity of human bone marrow and the prevalence of osteoblastic progenitors. J Orthop Res 2001;19:117-25.

20. Abramson SB, Amin A. Blocking the effects of IL-1 in rheumatoid arthritis protects bone and cartilage. Rheumatology (Oxford). 2002;41:972-80.

21. Kobayashi M, Squires GR, Mousa A, et al. Role of interleukin-1 and tumor necrosis factor alpha in matrix degradation of human osteoarthritic cartilage. Arthritis Rheum 2005;52:128-35.

22. Buckwalter JA, Mankin HJ, Grodzinsky AJ. Articular cartilage and osteoarthritis. Instr Course Lect 2005;54:465-80. 